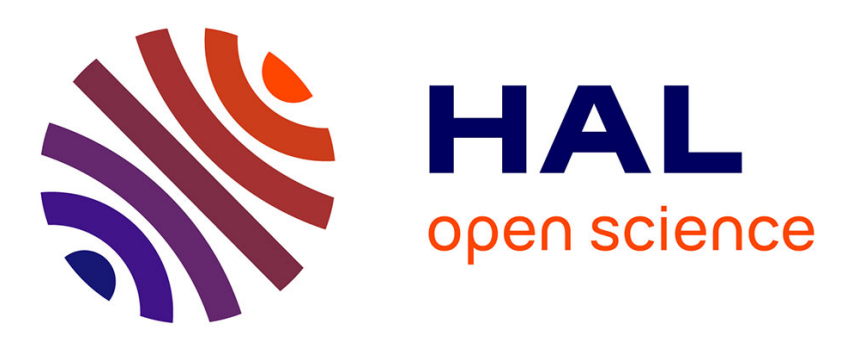

\title{
Joint source-channel coding: secured and progressive transmission of compressed medical images on the Internet
}

Marie Babel, Benoît Parrein, Olivier Déforges, Nicolas Normand, Jean-Pierre Guédon, Véronique Coat

\section{To cite this version:}

Marie Babel, Benoît Parrein, Olivier Déforges, Nicolas Normand, Jean-Pierre Guédon, et al.. Joint source-channel coding: secured and progressive transmission of compressed medical images on the Internet. Computerized Medical Imaging and Graphics, 2008, 32 (4), pp.258-269. 10.1016/j.compmedimag.2008.01.002 . hal-00273282

\section{HAL Id: hal-00273282 \\ https://hal.science/hal-00273282}

Submitted on 14 Apr 2008

HAL is a multi-disciplinary open access archive for the deposit and dissemination of scientific research documents, whether they are published or not. The documents may come from teaching and research institutions in France or abroad, or from public or private research centers.
L'archive ouverte pluridisciplinaire HAL, est destinée au dépôt et à la diffusion de documents scientifiques de niveau recherche, publiés ou non, émanant des établissements d'enseignement et de recherche français ou étrangers, des laboratoires publics ou privés. 


\title{
Joint source-channel coding: secured and progressive transmission of compressed medical images on the Internet
}

\author{
Marie Babel ${ }^{\mathrm{a}, *}$ Benoît Parrein ${ }^{\mathrm{b}}$ Olivier Déforges ${ }^{\mathrm{a}}$ Nicolas Normand ${ }^{\mathrm{b}}$ Jean-Pierre Guédon ${ }^{\mathrm{b}}$ \\ Véronique Coat ${ }^{\mathrm{a}}$ \\ ${ }^{\mathrm{a}}$ IETR UMR CNRS 6164 - Image and Remote Sensing Group / INSA of Rennes,20 av. des Buttes de Coësmes - CS 14315, \\ 35043 Rennes, France \\ ${ }^{\mathrm{b}}$ IRCCYN UMR CNRS 6597, Image and Videocommunications Team, La Chantrerie, rue Christian Pauc, BP 50609, 44306 \\ Nantes Cedex 3, France
}

\begin{abstract}
The joint source-channel coding system proposed in this paper has two aims: lossless compression with a progressive mode and the integrity of medical data, which takes into account the priorities of the image and the properties of a network with no guaranteed quality of service. In this context, the use of scalable coding, Locally Adapted Resolution (LAR) and a discrete and exact Radon transform, known as the Mojette transform, meets this twofold requirement. In this paper, details of this joint coding implementation are provided as well as a performance evaluation with respect to the reference CALIC coding and to unequal error protection using Reed-Solomon codes.
\end{abstract}

Key words: medical image compression; LAR method; Mojette transform; scalability; priority encoding system;

\section{INTRODUCTION}

Through the development of PACS (Picture Archiving and Communication Systems), health care systems have come to rely on digital information. Furthermore, future medical applications will have to integrate access to generalized databases that contain the personal medical information of each patient. Efficient image management consequently becomes a key issue in designing such a system. Given this situation, two main elements must be considered at the same time: compression and security strategies specific to image handling [1].

Naturally, teleradiology systems integrate the notion of security [2]. In particular, they must guaran-

\footnotetext{
* Corresponding author.

Email addresses: Marie.Babel@insa-rennes.fr (Marie Babel), Benoit.Parrein@polytech.univ-nantes.fr (Benoît Parrein).
}

tee the integrity (to prevent the alteration of data), authentication (to check the sender) and the confidentiality (to prevent unauthorized access) of the medical data at all times. The availability of the information can be ensured by the Internet Protocol (IP). Moreover, wireless transmissions play an increasingly important part in the achievement of this goal, [3-5] especially in the context of emergency medicine [6]. However, this access to information must be accompanied by security primitives. For privacy and authentication purposes, the traditional security solutions integrated in the DICOM standard cover encryption processes and digital signatures [7].

To the best of our knowledge, very few publications cover the loss of entire IP packets in medical data transmissions [8]. In a more general framework such as image transmission, most studies relate to the implementation of error control coding 
e.g. Reed-Solomon codes to compensate for packet loss by avoiding retransmissions [8][9]. By adjusting the correction capacities and, thus, the rates of redundancy, it is possible to adapt to both a scalable source and an unreliable transmission channel. This is the purpose of Unequal Error Protection (UEP) codes which are now mature and proposed in standardization processes [10]. The specific problem of medical image integrity is very often the volume of the data being transmitted (cf lossless coding, 3D$4 \mathrm{D}$ acquisition etc.). Within this framework, UEP must meet algorithmic complexity requirements to satisfy real time constraints. With this in mind, we propose in this paper the use of an exact and discrete Radon transform, the Mojette transform, to compensate for IP packet loss.

With regard to compression, archiving implies lossless compression. Indeed, lossless compression is required either from a legal point of view (relative to each country), or from the radiology point of view (to avoid any misdiagnosis) [11]. The most efficient lossless compression schemes, such as CALIC [12] or Edge Directed Prediction based solution [13], do not provide any progressive transmission of the information yet this can be useful for transmissions on low bitrate networks, in particular on the wireless channel. Few schemes are dedicated to medical image compression. Among them the SSM coder [14] outperforms other solutions on some particular classes of images (US images) at the expense of complexity (selection between several coders, successive optimization steps of many parameters for each image classes).

The DICOM standard dedicated to medical image compression, has recently integrated the JPEG2000 standard file format [15]. Preliminary work has indeed stressed the need for a compression method that provides both scalability and advanced functionalities such as Region Of Interest (ROI) coding [16]. The Interleaved $\mathrm{S}+\mathrm{P}$ algorithm, based on the LAR (Locally Adaptive Resolution) coding method [17], produces better coding results than the JPEG2000 lossless mode. Moreover, thanks to and "intelligent" scalable semantic representation of the image, it provides high scalability together with advanced services.

This paper describes combination of the two methods (the LAR scalable coding scheme and the Mojette transform) which offers an efficient algorithm for the secure and progressive transmission of compressed medical images. The idea is to use and to preserve the intrinsic hierarchy of the Inter- leaved $\mathrm{S}+\mathrm{P}$ coder. An UEP system is then built using the Mojette transform to provide protection against the loss of entire streams (IP packets) that represent essential information.

The structure of the paper is as follows. We begin by describing the LAR coding scheme and the associated semantic representation. The section 3 details the scalable Interleaved $\mathrm{S}+\mathrm{P}$ solution. In the section entitled 4, we develop the different coding modes that are available before moving on to present the Mojette transform is presented (section 5) and explaining the entire joint LAR-Mojette method (section 6$)$.

\section{LAR METHOD FOR LOSSY GREYSCALE IMAGE COMPRESSION}

The LAR method was first designed for the purposes of low bit-rate greyscale image coding [18]. Recent research has led to the construction of scalable encoding schemes based on original pyramidal predictive descriptions, that are efficient for both lossless and lossy compression purposes $[17,19]$. In this section, we describe the basic principles of the LAR scheme.

\subsection{Principles}

In general, image representation and image compression are two distinct topics in the image processing field. However, the LAR method tries to link these two processes. The concept has in fact two major benefits. First, good representation naturally leads to the building of a better coding scheme. Secondly, advanced functionalities such as Region Of Interest coding can be easily implemented.

The LAR compression method is a two-layer codec: a flat codec and a complementary spectral one. This follows the idea developed in particular in [20], where images are considered to be the superposition of global information and texture (local information). The flat LAR codec is designed to provide a low bit-rate compressed image, whereas the spectral one encodes the texture. The originality of the proposed method lies in the fact that our scheme provides a content-based image coding method.

The following two paragraphs describe these flat and spectral coders respectively. Note that the quality of the low resolution LAR image has already been evaluated and recognized to be better than JPEG2000 [21]. 


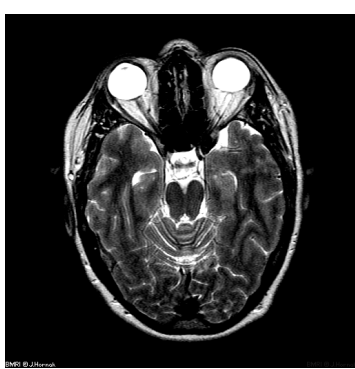

(a) Original image.

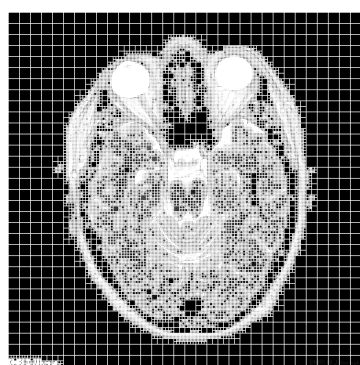

(b) Grid: 0.031bpp

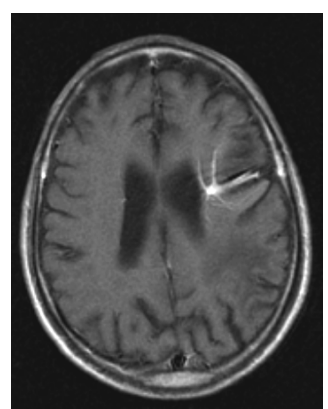

(c) Original image

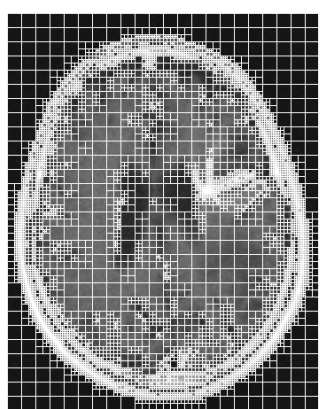

(d) Grid: 0.032bpp

Fig. 1. Visualization of the grid produced by the spatial coder of the LAR method.

\subsection{Flat coder}

The basic idea is that local resolution, in other words pixel size, can depend on local activity. This leads to the construction of a variable resolution image based on a quadtree data structure. The final size of blocks (typically from $16 \times 16$ to $2 \times 2$ ) is computed using an estimation of a local morphological gradient: each block is then reconstructed by its average luminance. Thanks to this type of block decomposition, their size implicitly gives the nature of the said block. Small blocks are naturally located on contours whereas large ones are situated in smooth areas. Discrimination between areas is clearly visible on figure 1.b and 1.d. In a lossy context, this image content information controls a quantization of the luminance that is in accordance with visual perception: large blocks require fine quantization (in uniform areas, human vision is strongly sensitive to brightness variations) while coarse quantization (low sensitivity) is sufficient for small blocks.

To sum up, this coder has two main distinctive characteristics. Firstly, it provides a very fast and efficient tool for high compression ratios, and secondly, the method simplifies the image source by removing the local texture while preserving object boundaries. Perceptible block artefacts in homogenous areas are easily removed by efficient post-processing: in particular, an adaptive interpolation based on the optimal recovery theory can be implemented [22].

For color images, another improvement was achieved by the representation of self-extracting regions. Natural extensions of this particular process have also made it possible to address medium and high quality encoding and the region-level encoding of chromatic images [23].

\subsection{Spectral coder}

The flat LAR coder is clearly dedicated to lowbit rate image coding. To obtain higher image quality, the texture (whole error image) can be encoded through the spectral coder (second layer of the LAR coding scheme) which uses a DCT adaptive blocksize approach [24]. In this case, both the size and the DC components are provided by the flat coder. The use of adapted block size naturally allows for a semantic scalable encoding process. For example, edge enhancement can be made by only transmitting the AC coefficients of small blocks. Further refinements can be envisaged by progressively sending larger block information.

\section{SCALABLE LOSSLESS CODING: INTERLEAVED $\mathbf{S}+\mathbf{P}$ METHOD}

In medicine, only the lossless coding of images is authorized for archiving purposes. The DICOM standard has recently integrated the JPEG-2000 standard file format [15]: it enables scalable coding of a picture together with Region Of Interest (ROI) encoding. A new method, Interleaved $\mathrm{S}+\mathrm{P}$ supported by the LAR concepts, is described in this section.

\subsection{General principles}

The "Interleaved $\mathrm{S}+\mathrm{P}$ " algorithm is designed to provide a lossless representation of images. This new method keeps the same main structure as the LAR method (presented in section 2), namely a two-layer codec, so that the first part of the compressed bitstream allows the reconstruction of the LAR block image. A major improvement lies in the fact that the 


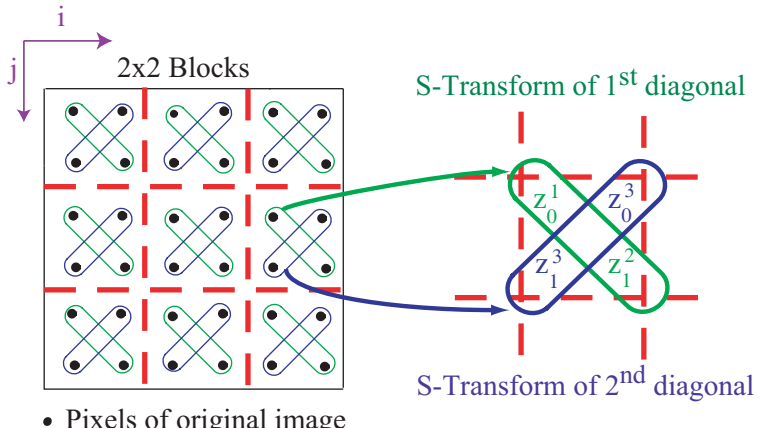

- Pixels of original image

Fig. 2. Original application of the S-Transform

pyramidal decomposition allows for a highly scalable transmission of compressed data.

LAR pyramidal decomposition is based on an adaptation of the $\mathrm{Wu}$ predictor [25]. For full resolution images, errors are coded by means of triple interlaced sampling of the original image. Consequently, we tend to obtain a spatial configuration of a $360^{\circ}$ type neighborhood surrounding a given pixel so that the resulting prediction error is drastically reduced.

The Interleaved $\mathrm{S}+\mathrm{P}$ algorithm is achieved in two main steps. The first stage consists of applying the 1D S-Transform on the 2 vectors formed by 2 diagonally adjacent pixels in a $2 \times 2$ block, as depicted in figure 2 .

In the following, $z_{0}$ and $z_{1}$ denote the Stransformed coefficients in such a way that if $\left(u_{0}, u_{1}\right)$ is a pair of values, we have:

$$
\begin{aligned}
& z_{0}=\left\lfloor\left(u_{0}+u_{1}\right) / 2\right\rfloor, \\
& z_{1}=u_{1}-u_{0} .
\end{aligned}
$$

The second step constitutes the prediction process. The prediction is in turn achieved in three successive passes. We also denote $z_{i}^{k}$, where $i \in\{0,1\}$ and $k \in\{1,2,3\}$ constitute the $z_{i}$ coefficient coded through the $k^{\text {th }}$ pass.

The first prediction pass uses a traditional DPCM system to encode a uniform subsampled image formed by the average of two diagonally adjacent pixels (first diagonal) within each $2 \times 2$ block $\left(z_{0}^{1}\right.$ transformed coefficients). Then the second pass predicts the $z_{1}^{2}$ transformed coefficients in order to reconstruct the value of the two pixels of the first diagonal, taking into account the fact that the mean value is already known (Fig. 3). At this stage, the $360^{\circ}$ type prediction consists of the already known values of the current pass and the diagonal means coded by the first pass.

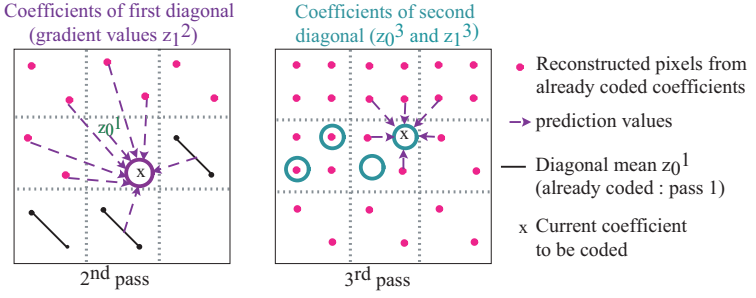

Fig. 3. Second and third pass of the transformed coefficients prediction process.

Finally, the third pass encodes the remaining half of the original image composed of the set of $z_{0}^{3}$ and $z_{1}^{3} \mathrm{~S}$-coefficients. Once again, thanks to the reconstructed pixels resulting from the two previous passes, a completely spatially enclosing and adjacent context is available to predict the transformed pixel.

\subsection{Pyramid construction - Interleaving}

In the previous paragraph, we described the basic elements of our algorithm. The Interleaved $\mathrm{S}+\mathrm{P}$ method simply results from the extension of this scheme. We define here the pyramid construction as well as its interleaving property. Let $J$ the original image be of size $N_{x} \times N_{y}$. The multiresolution representation of an image is described by the set $\left\{Y_{l}\right\}_{l=0}^{l_{\max }}$, where $l_{\max }$ is the top of the pyramid and $l=0$ the full resolution image. As an extension of the $\mathrm{Wu}$ method, four blocks $\frac{N}{2} \times \frac{N}{2}$ are gathered into one block $N \times N$ valued by the average of the two blocks of the first diagonal (first S-pyramid on fig. 4). This produces the following equation:

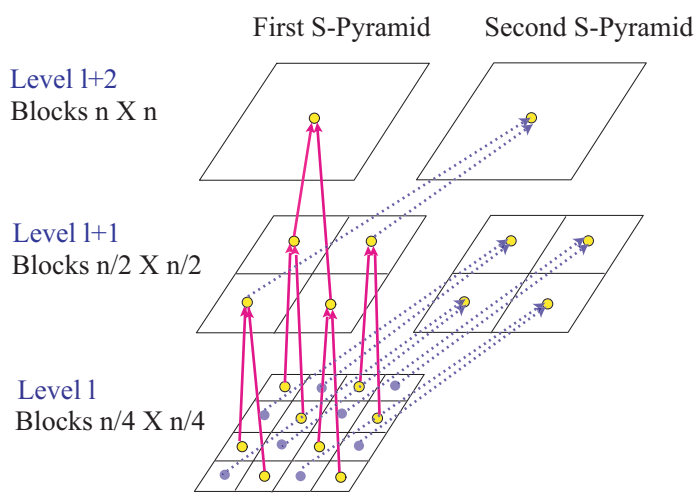

Fig. 4. Construction of the pyramid through two interleaved S-pyramids 


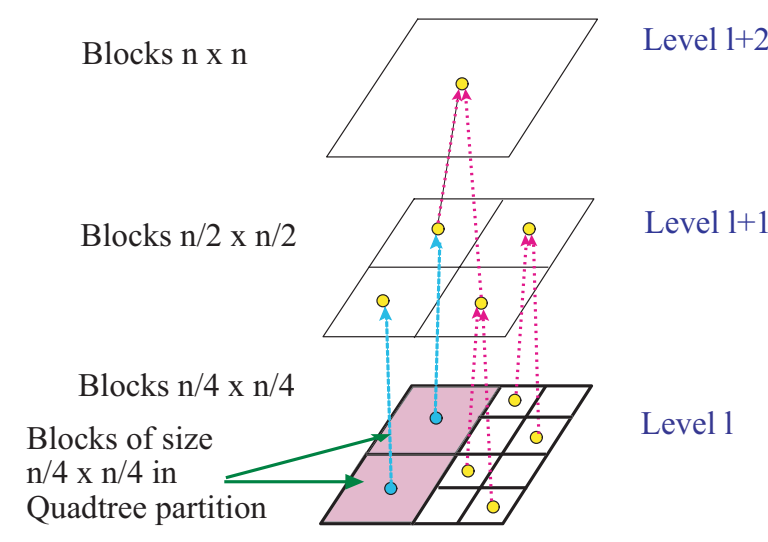

Fig. 5. Conditional decomposition: extraction of LAR block image data

$$
\begin{cases}l=0, & Y_{0}(i, j)=J(i, j) ; \\ l>0, & Y_{l}(i, j)=\left\lfloor\frac{Y_{l-1}(2 i, 2 j)+Y_{l-1}(2 i+1,2 j+1)}{2}\right\rfloor,\end{cases}
$$

with $0 \leq i \leq N_{x}^{l}, 0 \leq j \leq N_{y}^{l}$, where $N_{x}^{l}=N_{x} / l$ and $N_{y}^{l}=N_{y} / l$.

The transformation of the second diagonal of a given $2 \times 2$ block can also be seen as a second $\mathrm{S}$ pyramid, where the pixel values depend on the values existing on the lower level of the first S-pyramid. This specific construction of the algorithm can be interpreted as an interleaved process, with the second S-pyramid depending entirely on the first one.

\subsection{Interleaved $S+P$ Pyramid Decomposition - Refined prediction model}

The Interleaved $\mathrm{S}+\mathrm{P}$ pyramidal decomposition process results from the extension of the previously described prediction method to the whole pyramid. As mentioned in section 2, the decomposition is realized in two successive processes. The first one is aimed at obtaining the block image, while the second one enables the reconstruction of the texture information. The LAR specific grid is used for this purpose, as depicted in figure 5. An image denoted $\mathrm{Siz}$ is introduced: it contains the size information (the block size, i.e. from 2 to 16) worked out from Quadtree partition.

For a given level $l$, when only processing the pixels of size $\operatorname{Siz}(x \times l, y \times l) \leq 2^{l}$, the codec reconstructs the LAR low resolution image. This block image is then obtained after the first pyramidal decomposition. The second pyramidal decomposition then makes it possible to recover texture information and an estimation process is applied to pixels of size $\operatorname{Siz}(x \times l, y \times l)>2^{l}$. Implemented predictors differ according to:

- the nature of the coefficients $\left(z_{0}\right.$ and $\left.z_{1}\right)$,

- the location of pixels (first or second S-pyramid),

- the nature of local information, i.e. blocks image or texture.

\subsection{Compression results}

In [17] we have already emphasized the superiority of the Interleaved $\mathrm{S}+\mathrm{P}$ over the state-of-the-art lossless compression method in terms of first-order entropy for natural images. In this paragraph, we present some results on medical images ${ }^{1}$.

Table 1 shows the first-order entropy resulting from the application of Interleaved S+P. This entropy can be directly compared with the ones obtained when using the S+P [26] and CALIC [12] state-of-the-art methods. CALIC is considered to be the reference for lossless medical images compression in terms of performance [27]. Note that no entropy encoder was used here.

Clearly, our coder widely outperforms both $\mathrm{S}+\mathrm{P}$ and CALIC equivalents. The Interleaved $\mathrm{S}+\mathrm{P}$ coder presents an average entropy equal to $3.02 \mathrm{bpp}$, whereas CALIC and $\mathrm{S}+\mathrm{P}$ reach 3.20 and $3.16 \mathrm{bpp}$ respectively. The Interleaved $\mathrm{S}+\mathrm{P}$ coder provides its best results on mammograms and MRI images. In addition, our method offers an image encoder that is scalable in terms of both resolution and quality.

The performances of our algorithm stem from the initial quadtree partition. As it is perfectly correlated to the local activity, the method uses the information to adapt the prediction process in such a way as to decrease coding cost. Moreover, we split the bitstreams into different substreams according to the nature, location and local activity of the corresponding pixel. Implicit context modeling is then used, and this drastically reduces the final entropy.

This is particularly true when applied to medical images. Indeed, mammogram or MRI images typically contain two distinct areas: a large black zone (background) and a strongly textured area. The variable size block representation peculiar to LAR coders provides an efficient representation of these images and enables efficient compression of the information. For example, the prediction errors relative to large blocks (located in uniform areas) are

\footnotetext{
1 Tested images can be found on the following web site: http://eelmpo.cityu.edu.hk/imagedb/ and http://www. cis.rit.edu/htbooks/mri/inside.htm
} 


\begin{tabular}{|c|c|c|c|c|}
\hline \multirow[b]{2}{*}{ Image } & \multicolumn{4}{|c|}{ Entropy (bpp) } \\
\hline & Size & $\mathrm{S}+\mathrm{P}$ & CALIC & Int. $S+P$ \\
\hline colon & $512 \times 512$ & 3.53 & 3.33 & 2.87 \\
\hline angiot & $512 \times 512$ & 5.20 & 5.19 & 4.99 \\
\hline us & $512 \times 448$ & 3.78 & 3.60 & 3.55 \\
\hline angio & $512 \times 512$ & 3.11 & 3.10 & 3.10 \\
\hline $\mathrm{cr}$ & $1744 \times 2048$ & 3.30 & 3.30 & 3.27 \\
\hline echo & $720 \times 496$ & 3.28 & 3.41 & 3.44 \\
\hline x_ray & $2048 \times 1680$ & 2.39 & 2.27 & 2.36 \\
\hline mr_head_p & $256 \times 256$ & 4.27 & 4.26 & 4.08 \\
\hline mr_thorax & $256 \times 256$ & 4.60 & 4.71 & 4.61 \\
\hline ct_abdomen_fr & $512 \times 512$ & 2.61 & 2.54 & 2.61 \\
\hline ct_abdomen_tr & $256 \times 256$ & 3.43 & 3.38 & 3.43 \\
\hline mdb001 & $1024 \times 1024$ & 1.64 & 1.60 & 1.26 \\
\hline mdb005 & $1024 \times 1024$ & 2.19 & 2.13 & 1.80 \\
\hline mdb007 & $1024 \times 1024$ & 1.91 & 1.86 & 1.53 \\
\hline mdb023 & $1024 \times 1024$ & 2.11 & 2.04 & 1.83 \\
\hline an01 & $512 \times 512$ & 3.22 & 3.03 & 2.75 \\
\hline an02 & $512 \times 512$ & 3.13 & 3.19 & 3.30 \\
\hline hd01 & $512 \times 512$ & 3.23 & 3.17 & 2.61 \\
\hline ex03 & $512 \times 512$ & 3.88 & 3.88 & 3.97 \\
\hline Average & & 3.20 & 3.16 & 3.02 \\
\hline
\end{tabular}

Table 1

First-order entropy (bit/pixels) of the proposed Interleaved $\mathrm{S}+\mathrm{P}($ Int. $\mathrm{S}+\mathrm{P}$ ), CALIC and $\mathrm{S}+\mathrm{P}$.

much lower than the others, and the resulting entropy is quite low.

\section{FUNCTIONALITIES - MODES OF ENCODING}

\subsection{Near-lossless encoding}

Among the common encoding modes, the one known as "near-lossless" essentially consists of applying small quantization to result in undetectable errors. As Interleaved $\mathrm{S}+\mathrm{P}$ derives from the scalable extension of the basic LAR scheme, it appears that its ability to preserve natural content easily adapts quantization to human visual perception. For example, figure 6 shows the result of pyramidal decomposition where a quantization step equal to 4 is applied to contours $(2 \times 2$ blocks $)$ and a step equal to 2 is applied to flat areas. As no visual distortion

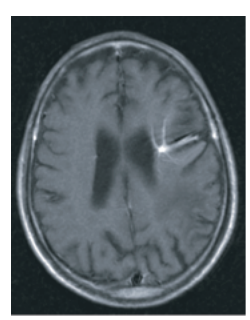

Original image

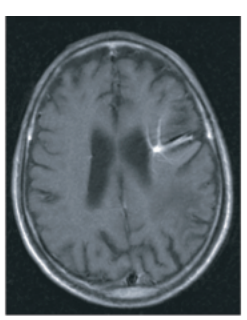

Near-lossless

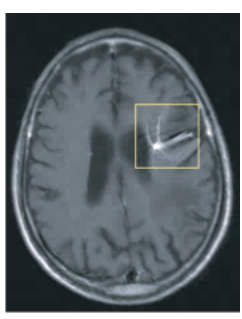

ROI
Fig. 6. Near-lossless encoded image (1.966 bpp) and example of the ROI selection.

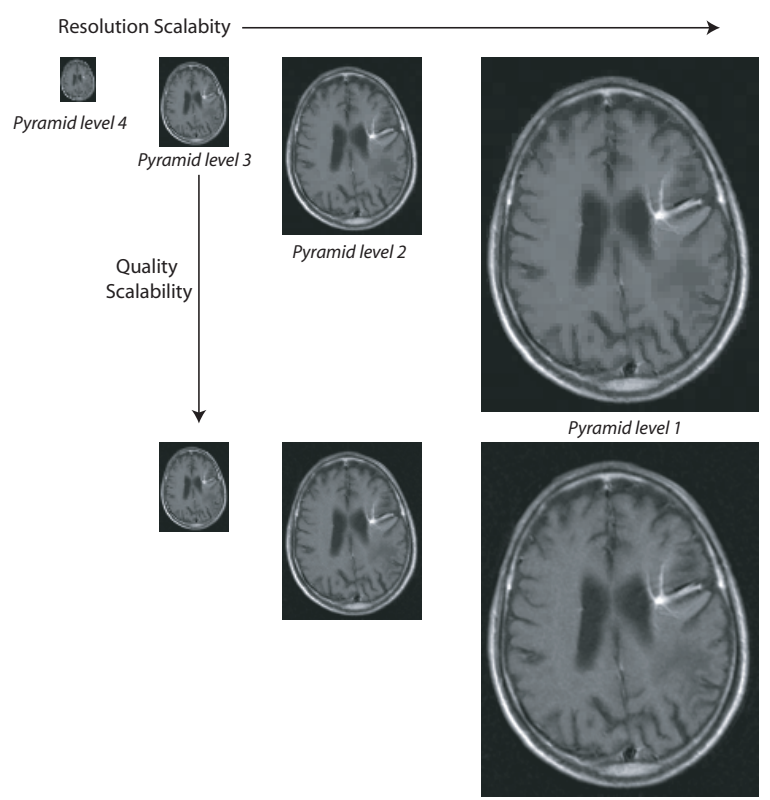

Fig. 7. Scalable representation of an image from level 4 to level 1 of the pyramid.

is noticeable, it is easy to imagine this functionality being used in the context of remote database access: the image is sufficient for initial medical reporting but the coding cost is lower.

\subsection{Scalable representation}

The pyramidal description of the images resulting from Interleaved $\mathrm{S}+\mathrm{P}$ encoding provides various scalability levels. The conditional decomposition (the constraint of two successive descent processes by the initial quadtree partition of the image) provides a highly scalable representation in terms of both resolution and quality. Figure 7 shows an example of this outstanding characteristic of our method and it is a major advantage for applications such as remote database consultation, teleradiology or other advanced client-server solutions. 
4.3. Self-extracting region representation - Region of interest

As mentioned above, the integration of JPEG2000 into the DICOM standard was also motivated by useful functionalities such as Region Of Interest coding. Typically, only a small portion of the medical image contains relevant and diagnostically useful information, while the remaining portion is displayed only as a visual background [28,27]. Through the definition of a Region of Interest (ROI), images can be lossly compressed overall and losslessly encoded locally. Combined with a progressive encoding scheme, region scalability allows faster access to significant data.

However, the JPEG-2000 ROI coding process is totally constrained by tile description. The LAR scheme enables more flexible solutions in terms of ROI shape and size. Indeed, an ROI can be simply described at both the coder and decoder as a set of blocks resulting from the quadtree partition. As the ROI is built from the variable block size representation, its enhancement (texture coding) is straightforward: it merely requires execution of the Interleaved $\mathrm{S}+\mathrm{P}$ codec for the validated blocks, i.e. ROI internal blocks. A simple example of this scheme is shown in figure 6. Unlike traditional compression techniques, the LAR low resolution image does not introduce strong distortions on the ROI contours. Such distortion usually makes the image too unreliable to be used.

Previous work centres on the definition of an original hierarchical self-extracting region representation based on the initial LAR partition [21]. This advanced scheme can be combined with the Interleaved $\mathrm{S}+\mathrm{P}$ coding technique to introduce another level of scalability. In addition to the pyramidal representation described in section 3 , this hierarchical representation of regions provides a highly scalable scheme with a wide spectrum of services. Figure 8 shows an example of ROI encoding. In it, a ROI is represented by a set of regions obtained through our self-extracting region representation process.

\section{ERROR RESILIENCE CODING BY MOJETTE TRANSFORM}

In this section, we take the process of LAR coding for transmission purposes a little further and propose a reliable communicating multimedia system. In addition to the heart of the compression ma-

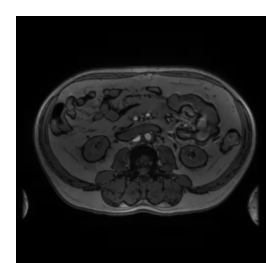

(a)

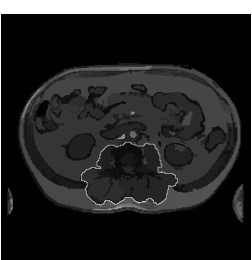

(b)

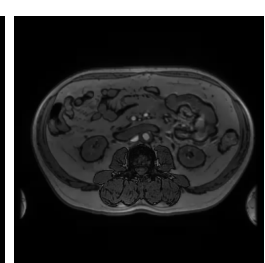

(c)
Fig. 8. ROI coding using the self-extracting region representation. (a) LAR low resolution image (first descent, 0.55 bpp) - (b) 124 regions representation - (c) background encoded at low bit rate, ROI losslessly encoded

chinery, we build unequal error protection coding depending on the sensitivity of each portion of the source coding stream. Roughly speaking, the most important part of the image is more protected by redundant information than non significant data. Fine granularity can be obtained for good adaptation both to the hierarchy of the image and to the channel properties that we define in this paper as joint source channel coding.

A great deal of research work has been done in this area over the past decade. The working draft of JPEG2000 WireLess (JPWL)[10] proposes concentrated unequal protection on the main header and the tile header with the characteristic that any error on these part of the stream is fatal for decoding. Error sensitivity is characterized by the PSNR. Conventional Reed-Solomon error correction codes are applied to a symbol level to provide protection. In [9], the codes $(160,64),(80,25)$ and $(40,13)$ are chosen and given redundancy rates of between $150 \%$ and $220 \%$ with some 500 bytes for headers. This very strong protection obviously improves the chances of success in decoding when binary losses occur but it also guarantees the integrity of the headers whether the properties of the channel are good or very bad. Furthermore, performance evaluation and protection on a symbol level are far removed from the real channels like wireless channels as can be seen for example through the variations in the protocol IEEE802.xx (WLAN or WiMax). More precisely, the approach never considers the effectiveness of the mechanisms operated on the level of Media Access Control (MAC) layer and physical (PHY) layer such as the hybrid ARQ (Automatic Query Request) combining efficient channel coding (turbo-code) and retransmission. Likewise, the working draft does not consider the exploratory research carried out over the past ten years on unequal error protection [29] 
or the new representations based on a multiple description of information [30].

In this section, we consider the PHY and MAC layers as effective to deliver true symbols so as to focus all our attention of unequal protection at the transmission unit level i.e the packet level. Protection is provided for the whole LAR coding stream. It depends on the sensitivity of each substream and the packet loss rate. The core joint source channel coding uses the Mojette transform.

\subsection{The direct Mojette transform}

The Mojette transform [31] is an exact and discrete Radon transform. It may seem strange that we should be using a conventional medical imaging tool for image communication but it easily describes an image in terms of a finite set of 1D-projections that will be our transport units in the following section of the article. Each angle of projection $\theta$ is defined as a couple of integers $(p, q)$, relatively primes, with $\frac{q}{p}=\tan \theta$. The direct Mojette transform of an image $f(k, l)$, denoted by $\mathcal{M} f$, represents a set of $N$ projections $\mathcal{M}_{p, q} f$ such as

$$
\mathcal{M} f=\left\{\operatorname{proj}_{p_{i}, q_{i}}, i=1,2 \ldots N\right\} .
$$

To compute this transform, we simply use additions in directions determined by the couple of integers $(p, q)$. The resulting projections are formed by a set of elements called bins. The value of a projection bin $m$ is calculated by the sum of pixels $f(k, l)$ located on the line determined by $m=-q k+p l$. The following equations stand for the definition of the Mojette transform:

$$
\operatorname{proj}_{p_{i}, q_{i}}(m)=\sum_{k} \sum_{l} f(k, l) \Delta(m+k q-p l),
$$

where $\Delta$ is the Krönecker function ${ }^{2}$.

Since each pixel contributes to one bin, the order of complexity is necessarily $\mathcal{O}(I)$ for any projection and for $I$ pixels. If we want to compute a $N$ projections set, the order of complexity is $\mathcal{O}(I N)$, linear with the number of pixels and with the number of projections.

The Mojette transform is linear with the number of pixels and the number of projections, in the same way as the Fourier and Meyer (wavelet) transforms. Unlike them, however, Radon and Mojette

\footnotetext{
$2 \Delta(m)= \begin{cases}1 & \text { if } m=0 \\ 0 & \text { otherwise }\end{cases}$
}

transforms do not aim to obtain an orthogonal decomposition of the signal; they are designed to obtain a frame. They produce useful redundancy for reconstruction. In fact, we consider this to be the fundamental difference between these two families of transforms: while the Fourier and Meyer transforms provide a hierarchical order of coefficients, the bins resulting from the application of Radon and Mojette transforms to a signal carry all the same weight. However, a given projection carries a quantity of information that is relative to its capacity to recover a portion of the original area. Moreover, each element contains the mean value (the sum of all projections bins), whereas in the case of orthogonal transform, this information is always contained in the first coefficient, but absent in the others.

This fundamental property of frames is a very useful feature for the direct and inverse implementation of Mojette transforms, indicating that the user can be left to choose the order of construction and reconstruction. The frame-like definition allows redundancies that can be further used for image description and image communication.

\subsection{The inverse Mojette transform}

\subsubsection{Algorithm}

The Mojette reconstruction algorithm is based on the fact that not all bins correspond to the same number of projected pixels. It is obvious that the value of a bin corresponding to a single pixel is identical to the pixel value. When such a one-to-one correspondence is found, the pixel value is copied from the bin value. The pixel is then removed from all the projections i.e. and its value is subtracted from all the bins where it projects. At this stage, the remaining bins represent the Mojette transform of the unreconstructed part of the image.

Reconstructing the image from a set of projections is then a process that iteratively:

(i) finds a reconstructible bin i.e. a bin projected from a single pixel,

(ii) "backprojects" its value onto the origin pixel,

(iii) updates the projections,

until the reconstruction is completed or no one-toone correspondence between a bin and a pixel is found.

\subsubsection{Reconstruction criterion}

A rectangular image $P \times M$ can be reconstructed from a set of projections with directions $\left\{\left(p_{i}, q_{i}\right)\right\}$ if 
and only if:

$$
\left\{\begin{array}{l}
\sum_{i}\left|p_{i}\right| \geq P \\
\text { or }\left|q_{i}\right| \geq M
\end{array} .\right.
$$

When building a set of reconstructible projections, we can choose to comply with either of the two conditions. For example, if we set all $q_{i}$ to 1 , a $P \times M$ image will require exactly $M$ projections to be reconstructed whatever its width $P$. From this reconstructible set of $M$ projections, we can build a redundant set by adding new projections. With $N-M$ extra projections, any subset of $M$ projections among the total $N$ is enough to reconstruct the image.

\subsection{Multiple description by Mojette transform}

Within the framework of communication systems, the Mojette transform can be applied to multiple description (MD) codes. These codes are precisely overviewed in [32]. MD code consists of separating an original source by means of several independent descriptions which give a degraded but useful version. Making descriptions individually good, yet not too similar, is the fundamental tradeoff of MD coding [32]. The Mojette transform can be simply used to realize this tradeoff [33]. We describe here the mechanism of the packetization strategy that converts the prioritized multiresolution (or layered) coding into an $\mathrm{N}$ unprioritized description using the Mojette transform.

Each element of a source bitstream is allocated to a 2D support which can be considered as a geometrical buffer in our communication system. Application of the direct Mojette transform to this buffer produces the descriptions. Each description in the MD stream fills an entire network packet: the terms "descriptions", "packets" and "projections" are thus used indifferently. The method, which uses several geometrical buffers, is described even if these buffers can be concatenated in order to optimize the stretch factor of the code i.e. the transmitted and original information ratio [34]. However, the separated design builds the protection scheme with a simpler partition of the data. It is based on separable geometrical buffers for each stream and priority. The Mojette transform of each support is then simultaneously computed. The number of necessary projections indicates the priority level supported by a buffer. Let $\rho$ be the protection function that maps each geometrical buffer to a number of projections i.e. $\rho_{i}$ projections are necessary to reconstruct the $i^{\text {th }}$ buffer.

Figure 9 gives an example of protection. Three rectangular supports have distinct reconstruction properties to obtain three scalable protection levels. Sub-streams 1, 2 and 3 are allocated respectively to geometrical buffers 1, 2 and 3. Support 1 (resp. 2 and 3 ) is reconstructible by means of two projections (resp. three and four) taken from the projection set $S=\{(-2,1),(-1,1),(0,1),(1,1)\}$. For this example, $\rho=\{2,3,4\}$. Packet 1 then contains all projections of angle $(0,1)$ whereas packet 4 contains projections of angle $(-2,1)$. This involves variable sizes of packets while simplifying the insertion of angle labels.

This method presents a solution to the need to protect scalability. The Mojette transform produces descriptions which are equivalent for the transmission network. For any given priority level, if the transmission is composed of $M$ projections and $N-$ $M$ redundant projections, it is possible to rebuild the layer in a deterministic way, starting with any $M$ packets received from among $N$. Using this mechanism, we have supported the hierarchy of the source over a best-effort channel.

\subsection{Optimal redundancy allocation}

In a simple manner, the Mojette transform can provide a redundant description of initial information. The purpose of optimal redundancy allocation is to determine the number of redundant projections according to the description of error sensibility and the probability of receiving the original information. In the case of a scalable source composed of hierarchical substreams, optimisation gives the overall number of sufficient projections for each substream: the protection function $\rho$.

Let $s$ be the substream index going from 1 to $L$ where $\mathrm{L}$ is the number of resolutions (or quality layers). The progressive reconstruction of the source assumes that substream $s$ is usable only if substream $s-1$ is rebuilt. It is not useful to provide greater protection for substream $s-1$ than for substream $s$. The $\rho$ function is thus monotonically increasing:

$$
\rho_{0}=0 \leqslant \rho_{1} \leqslant \rho_{2} \ldots \leqslant \rho_{L}
$$

Let $Q_{s}$ be the quality measurement associated with the image reconstructed from substreams 1 to $s$. Given the source scalability, we suppose $Q_{s} \geqslant$ $Q_{s-1}$. We can define a quality increment easily, $\Delta Q_{s}$, positive or null by: 


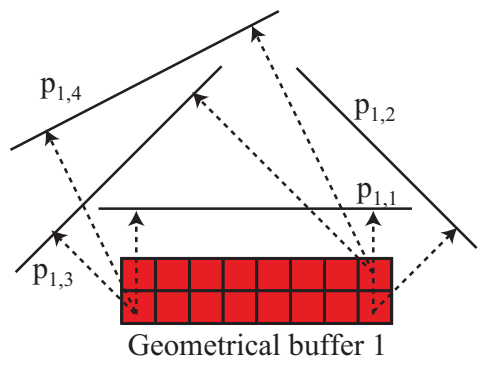

Geometrical buffer 1

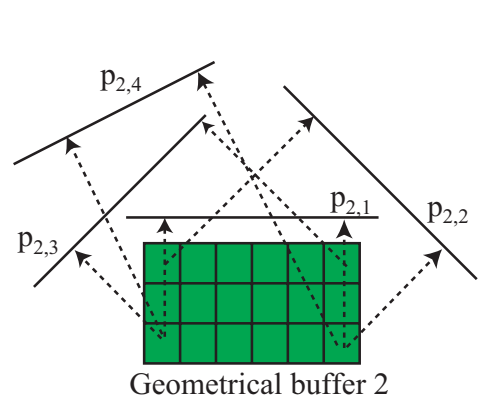

Geometrical buffer 2

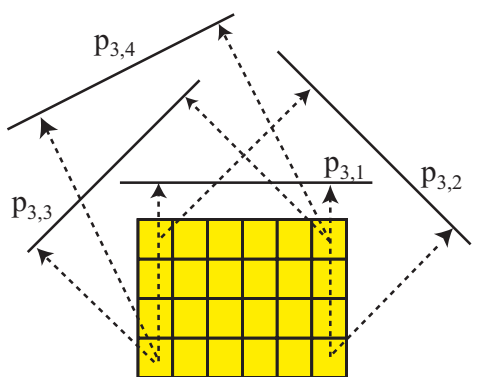

Geometrical buffer 3

Geometrical buffer projections

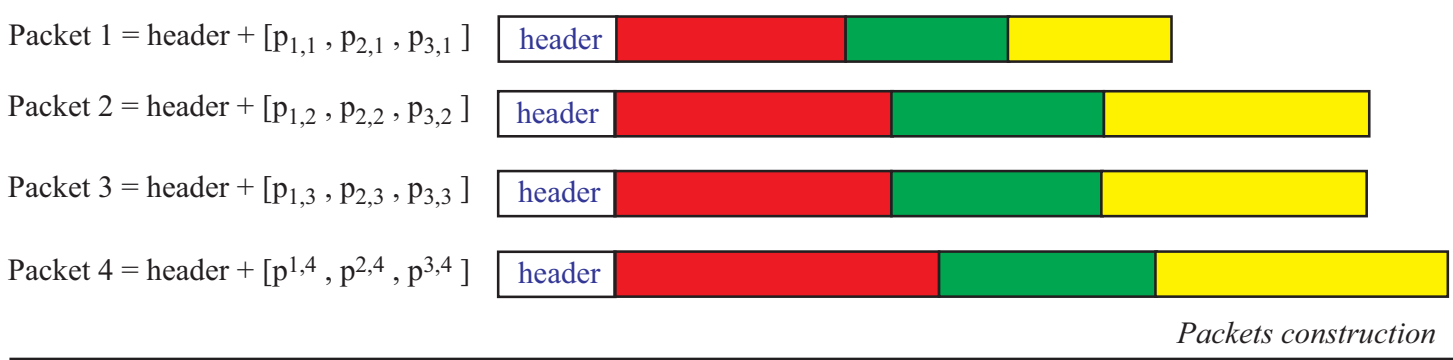

Fig. 9. Three-level protection scheme with geometrical buffer separation. One packet construction is realized by concatenating the same angle projections. $p_{a, b}$ stands for the $b^{t h}$ projection of the $a^{t h}$ geometrical buffer.

$$
\Delta Q_{s}=Q_{s}-Q_{s-1},
$$

where $\Delta Q_{0}=Q_{0}$. Let $X$ be the random variable representing the number of received projections. The optimal $\rho$ function maximizes the expected quality $E[Q]$ at the decoding stage:

$$
E[Q]=\sum_{s=0}^{L} Q_{s} P\left[\rho_{s} \leqslant X<\rho_{s+1}\right] .
$$

If $Q_{s}=\sum_{j=0}^{s} \Delta Q_{j}$,

$$
\begin{aligned}
E[Q] & =\sum_{s=0}^{L}\left(\sum_{j=0}^{s} \Delta Q_{j}\right) P\left[\rho_{s} \leqslant X<\rho_{s+1}\right], \\
& =\sum_{j}^{L} \sum_{s=j}^{L} \Delta Q_{j} P\left[\rho_{s} \leqslant X<\rho_{s+1}\right], \\
& =\sum^{j} \Delta Q_{j} \sum_{s=j}^{L} P\left[\rho_{s} \leqslant X<\rho_{s+1}\right], \\
& =\sum_{j=0}^{j \underline{L}_{0}} \Delta Q_{j} P\left[X \geqslant \rho_{j}\right] .
\end{aligned}
$$

From a practical point of view, the algorithm considers the $E[Q]$ change for each enable redundant value. Initially, no substream is transmitted: the protection level is equal to $N+1$. The number of projections required to rebuild each substream is then decremented. This increases the protection and, therefore, the global rate. The expected quality corresponding to unequal protection for a given channel loss profile is calculated for each protection level.

The maximisation of expected quality constitutes the link between source and channel coding by jointly considering the properties of the source (quality increments) and the feedback from the channel (reception probabilities).

\section{JOINT LAR AND MOJETTE IMAGE CODING}

Image transmission applications need compression and secured transmission of sensitive data simultaneously. The securization process is applied here to preserve the integrity of the data. In some ways, the securization process can also preserve the progressive mode provided by the source coding. This section presents the results of the joint source channel image coding implemented by LAR and Mojette image coding.

\subsection{Application to image transmission}

The general joint coding scheme is represented in figure 10. Our application relates to the transmis- 
sion of the test image 'Angiot' $(512 \times 512,8$ bits $)$. For that purpose the LAR compression scheme is used together with a simple arithmetic coder of zero order. An exponential distribution is retained for packet loss in order to validate our approach in the memory channel environment. A simple PSNR is used as a measurement of quality.

Lossless LAR coding is applied to the test image. The total rate is $5.05 \mathrm{bpp}$ which can be divided into 6 substreams corresponding to 6 major steps of the Interleaved $\mathrm{S}+\mathrm{P}$ compression. The first substream integrates both encoded data corresponding to the grid computed by the spatial coder, and the information resulting from level 4 of our multiresolution representation. Streams 2, 3 and 4 encode the estimation errors obtained during the first pyramidal decomposition for level 3,2 and 1 of the pyramid respectively. At this stage, the reconstructed image corresponds to the LAR low resolution image. Adding bitstream 5 (texture information) recovers the half-resolution image (level 1 , second pyramidal decomposition). Finally, bitstream 6 encodes the full resolution information losslessly. In this way, these six substreams correspond to the granularity of the protection where unequal redundant rates are allocated. Table 2 summarizes the properties of each substream in terms of size and quality increments.

Table 2

Properties of the LAR substreams for the test image "Angiot" $(512 \times 512)$.

\begin{tabular}{|l|c|c|c|c|c|c|}
\hline Substream & 1 & 2 & 3 & 4 & 5 & 6 \\
\hline $\begin{array}{l}\text { Size of layers } \\
\text { (kbytes) }\end{array}$ & 1.97 & 1.91 & 7.04 & 24.05 & 30.32 & 100.05 \\
\hline $\begin{array}{l}\text { Source rate } \\
\text { (w/o protec- } \\
\text { tion) (bpp) }\end{array}$ & 0.06 & 0.12 & 0.33 & 1.07 & 1.99 & 5.05 \\
\hline $\begin{array}{l}\text { Quality incre- } \\
\text { ments } s(\mathrm{~dB})\end{array}$ & 23.05 & 2.00 & 0.92 & 2.95 & 1.09 & 20.00 \\
\hline
\end{tabular}

From this scalable description of the source, we carry out the optimal redundancy allocation described at $\S 5.4$, taking into account the increments in quality and the exponential loss profile.

Unequal protection is then applied to the entire stream (header and image data). In case of a geometrical Mojette code, it is characterized by the number of sufficient projections chosen from among the number $N$ of sent projections. $N$ is set here to 16 projections.

Figure 11 summarizes the rate distortion analysis for two joint source-channel coding : a reference one using Maximum Distance Separable (MDS) codes

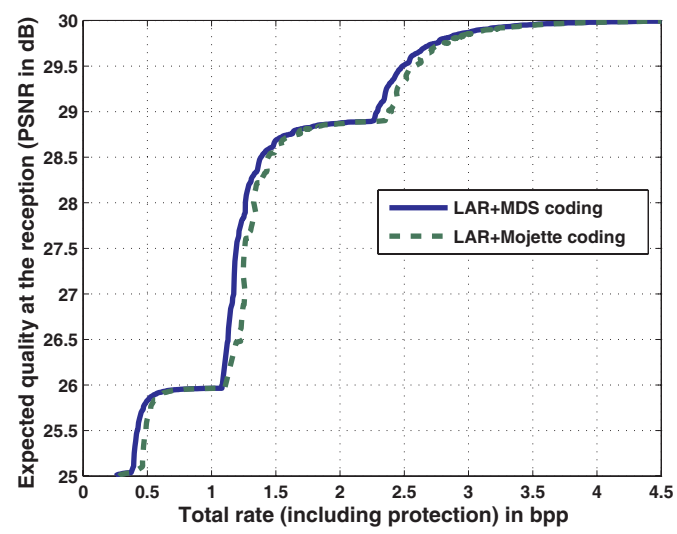

Fig. 11. Rate/quality analysis of joint source channel coding for memory channel (mean packet loss of 10\%) and PSNR measurement. The output rate includes LAR coding and MDS or Mojette protection. Three singularities correspond to the decisions to transmit refinement streams significantly improving the expected quality.

to which the Reed-Solomon codes belong and our scheme using Mojette transform. The analysis is performed in the lossy area for a mean packet loss equal to $10 \%$. The three singularities observed correspond to the decision of the protection system to transmit an additional substream in order to achieve the target quality.

For example, an expected quality of $27.00 \mathrm{~dB}^{3}$ can be obtained by the protection function $\rho=$ $\{9,11,14,16,17,17\}$. The last two substreams are not transmitted here to reach this target. The size of projections is equal to 2400 bytes on average for an overall joint source channel coding rate equivalent to $1.172 \mathrm{bpp}$. This rate can be compared to the original LAR rate (1.07 bpp), giving a redundancy rate of $9 \%$. This rate allows decoding even if the first packets are loss.

In a second example, the channel could provide a guaranteed and fixed bandwidth. In the lossless area, which is not given in the curve, if a redundancy rate of $6 \%$ is accepted for protection, i.e. an overall rate of $5.34 \mathrm{bpp}$, the lossless quality is expected thanks to the protection function $\rho=\{8,10,14,14,15,16\}$. In this example, the loss of half of the transport units is compensated for in order to rebuild stream 1 (the most important part of the LAR coding). In this case, all substreams are transmitted.

\footnotetext{
3 The difference between resolutions explains the low level of PSNR.
} 


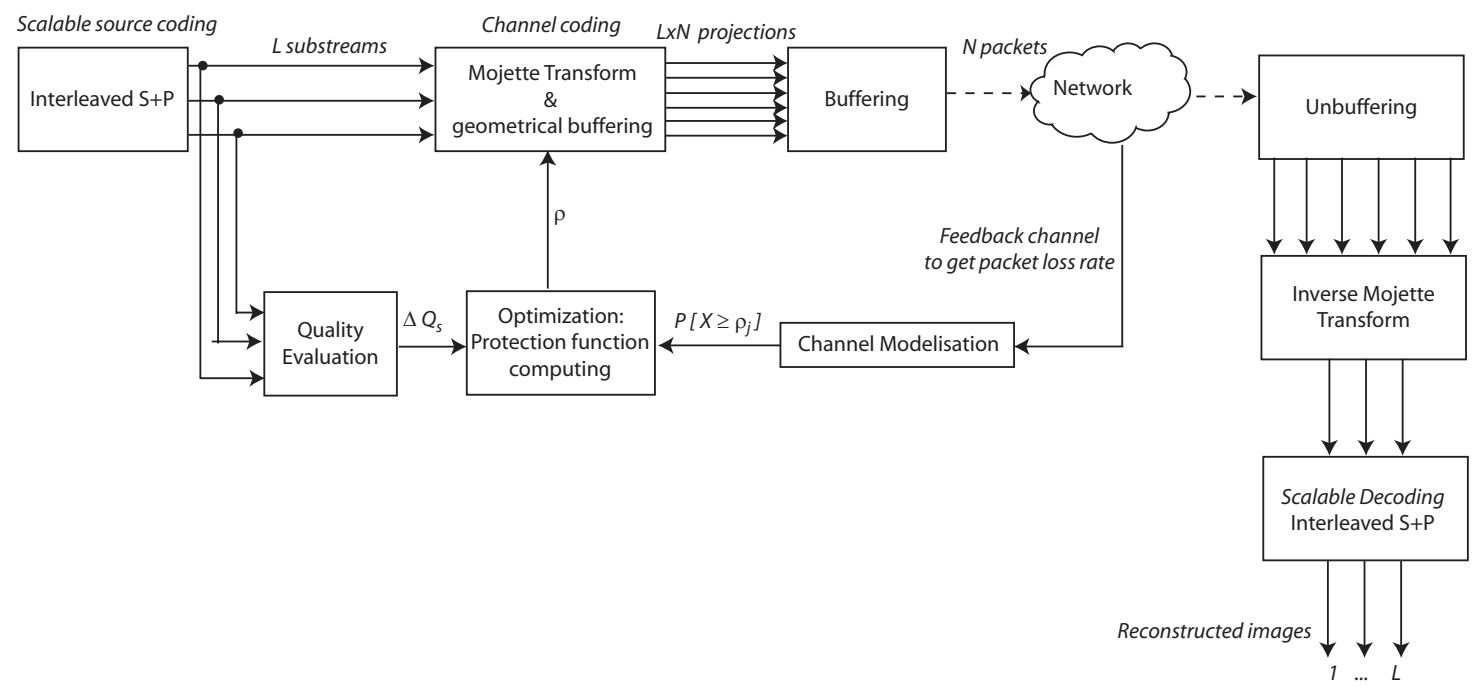

Fig. 10. General joint LAR-Mojette coding scheme.

\subsection{Performances}

The low density of parity checks allows fast iterative decoding by the inverse Mojette transform. Critical substreams are naturally rebuilt first. Contrary to original LAR coding, the loss of the first projections is not prohibitive for decoding. Degradations are progressive whatever the position of erasure in the image flow. Projections have the same rebuilding capacity. Consequently, our method bears a resemblance to the MDS. For these coding systems, any $\mathrm{M}$ code words selected from among the $\mathrm{N}$ transmitted are enough to rebuild the message. The system presented is of the $(1+\varepsilon)$ MDS type where $\varepsilon$ represents the necessary overhead for decoding. In the example depicted figure 11, the average overhead is about $2.78 \%$. This overhead can be reduced when mean packet loss increases. At 20\%, the overhead is $2.45 \%$ even if the protection increases. Geometrically speaking, if source rates are the same, the length of the geometrical buffer increases as protection reducing overhead. For infinite geometrical buffers, Mojette transform has MDS property. Typically, $\varepsilon$ is about $2 \%$ for the transmission of a $512 \times 512$ image. But the complexity is linear with the number of information elements, $I$, and the number of projections, $N$, both for encoding and decoding whereas MDS decoding presents a complexity in $\mathcal{O}\left(I \log ^{2} I\right)$ [35]. In addition, the transform offers greater flexibility given that MDS codes are strongly constrained by the algebra of the Galois fields. So the Mojette transform more efficiently supports the highly gran- ular hierarchy delivered by LAR or any scalable coding.

Performances are also distinguishable when comparing the unequal error protection scheme (UEP) with the equal error equivalent (EEP). In packet transport mode, the use of forward error correcting (FEC) codes like Reed-Solomon (RS) codes authorizes the loss of units without compromising the transmission of the information. Their correction capacity for the whole stream can, in certain cases, include the following property: it is possible to rebuild the original stream in a deterministic way as soon as any $M$ packets from among $N$ are received. That describes the EEP: one equal level of redundancy for the whole stream. Decoding fails if more than $N-M$ packets are lost. When exact rebuilding is impossible, it can be preferable to compensate for the errors rather than to correct them. This is the worthwhile feature of UEP which can deliver a degraded version when transmission is particularly disturbed.

Previous works have shown that the use of a lossily compressed image remains still an interesting feature for medical image purposes. As soon as the inherent distortions do not too much deteriorate the visual perception of the image, lossy compression appears as a suitable solution for telemedicine systems [36]. Thanks to our adapted UEP strategy, our scheme assures a quality of service in terms of image quality, even in case of hard conditions of transmission: the reconstructed images can be exploited by medical experts for diagnosis purposes. 


\section{CONCLUSION}

The LAR image coding method is a new scalable method which is not only efficient in terms of image quality for low bit-rates but also achieves an excellent compression ratio for losslessly encoded images. Each bitstream is associated with both a particular resolution level of the pyramid and a specific type of information (smooth areas or contours). This being so, losing a given bitstream leads to the reconstruction of the image at a limited resolution level. The LAR coder has the advantage of recovering low bitrate encoded images with good visual quality (e.g. no blocks or ringing effect).

The Mojette transform is then applied to each compressed bitstream provided by the LAR coder, providing hierarchical cost-controlled protection. All the projections and added redundancies are dispatched among the whole set of IP packets. Thus, as the cost of a Mojette transform only depends on the number of packets received and not on their contents, the decoder immediately launches a reconstruction process upon reception of each packet.

An original joint source-channel coding, based on LAR compression and Mojette transform, has been especially designed for Internet transmissions. In the application, a LAR encoder feeds data to the Mojette coder which behaves as a priority encoding system. The use of Mojette transforms to link the LAR to the priority encoding system provides a guarantee of end-to-end Quality of Service.

\section{References}

[1] R. Norcen, M. Podesser, A. Pommer, H.-P. Schmidt, A. Uhl, "Confidential storage and transmission of medical image data," Computers in Biology and Medicine 33 (3) (2003) 277-297.

[2] S. Sneha, A. Dulipovici, "Strategies for Working with Digital Medical Images," in: HICSS'06: Proceedings of the 39th Annual Hawaii International Conference on System Sciences, Vol. 5, 2006, p. 100.1.

[3] P. C. Pedersen, D. Sebastian, "Wireless Technology Applications in a Rural Hospital," in: 2004 ATA Annual Meeting, 2004.

[4] C. Pattichis,

E. Kyriacou, S. Voskarides, M. Pattichis, R.Istepanian, C. Schizas, "Wireless Telemedicine Systems: An Overview," IEEE Antennas and Propagation Magazine 44 (2) (2002) 143-153.

[5] J. Vucetic, "Telemedicine: The Future of Wireless Internet Applications," in: Southeast Wireless'03, 2003.

[6] N. Hashmi, D. Myung, M. Gaynor, S. Moulton, "A sensorbased, web service-enabled, emergency medical response system," in: EESR'05: Proceedings of the 2005 workshop on End-to-end, sense-and-respond systems, applications and services, 2005, pp. 25-29.

[7] H. Oosterwijk, "The DICOM standard, overview and characteristics," Ringholm Whitepapers (March 2004).

[8] A. Mohr, E. A. Riskin, R. E. Ladner, "Unequal Loss Protection : Graceful degradation of image quality over packet erasure channels through forward error correction," Journal on Sel. Areas in Com. 18 (6) (2000) 819-828.

[9] F. Dufaux, D. Nicholson, "JWL: JPEG 2000 for wireless applications," in: SPIE Proc. Applications of Digital Image Processing XXVII, Vol. 5558, SPIE, 2004, pp. 309318.

[10] JPEG. Editors, "JPEG 2000 image coding system - Part 11: Wireless JPEG2000 Committee Draft," in ISO/IEC CD 15444-11 / ITU-T SG8.

[11] P. White, "Legal issues in teleradioogy - distant thoughts!," The British Journal of radiology 75 (891) (2003) 201-206.

[12] X. Wu, N. Memon, "Context-based, adaptive, lossless image codec," IEEE Trans. On Communication 45 (4) (1997) 437-444.

[13] X. Li, M. T. Orchard, "Edge-Directed Prediction for Lossless Compression of Natural Images," IEEE Transactions on Image Processing 10 (6) (2001) 813-817.

[14] A. Pzrelaskowski, "Lossless Encoding of Medical Images: Hybrid Modification of Statistical Modelingbased Conception," Journal of Electronic Imaging 10 (4) (2001) 966-976.

[15] DICOM (Digital Imaging and Communications in Medicine), PS 3.5 Part 5: Data Structures and Encoding, NEMA, Rosslyn VA (May 2004).

[16] D. H. Foos, E. Muka, R. M. Slone, et al., "JPEG 2000 compression of medical imagery," in: SPIE Medical Imaging'00 - PACS Design and Evaluation: Engineering and Clinical Issues Vol. 3980, 2000, pp. 85-96.

[17] M. Babel, O. Déforges, J. Ronsin, "Interleaved S+P Pyramidal Decomposition with Refined Prediction Model," in: IEEE International Conference on Image Processing, ICIP'05, Vol. 2, Genova,Italy, 2005, pp. 750753.

[18] O. Déforges, J. Ronsin, "Nonuniform Sub-Sampling using Squares Elements : a Fast Still Image Coding at Low Bit-Rate," in: International Picture Coding Symposium PCS'99, Portland, Oregon, 1999.

[19] M. Babel, O. Déforges, J. Ronsin, "Lossless and Lossy Minimal Redundancy Pyramidal Decomposition for Scalable Image Compression Technique," in: 4th IEEE International Conference on Multimedia and Expos, ICME'03, Vol. 3, Baltimore, USA, 2003, pp. 161-164.

[20] B. Meyer, P. Tisher, "TMW : a new Method for Lossless Image Compression," in: Proc. Picture Coding Symposium, Berlin, 1997, pp. 533-538.

[21] O. Déforges, J. Ronsin, "Region of Interest Coding for Low Bit-Rate Image Transmission," in: Proc. International Conference on Multimedia and Expo ICME'2000, Vol. 1, 2000, pp. 107-110.

[22] D. D. Muresan, T. W. Parks, "Optimal Recovery Approach to Image Interpolation," in: Proc. IEEE ICIP'01, Vol. 3, Greece, 2001, pp. 848-851

[23] O. Déforges, J. Ronsin, "Supervised Segmentation at Low Bit Rates for Region Representation and Color 
Image Compression, in: IEEE International Conference on Multimedia and Expo," ICME'2002, Vol. 1, 2002, pp. 665-668.

[24] C. Chen, "Adaptive transform coding via quad-tree based variable block-size DCT," in: ICASSP'89, 1989, pp. 1854-1856.

[25] X. Wu, N. Memon, K. Sayood, "A Context-based, Adaptive, Lossless/Nearly-Lossless Coding Scheme for Continuous-Tone Images (CALIC)," International Standards Organization working document, ISO/IEC SC29/WG 1/N256.

[26] A. Said, W. Pearlman, "Reversible Image Compression via Multiresolution Representation and Predictive Coding, in: Visual Communication and Image Processing," Vol. 269, SPIE, 1993, pp. 664-374.

[27] D. A. Clunie, "Lossless Compression of Grayscale Medical Images: Effectiveness of traditional and state-oftheart Approaches," in: SPIE Medical Imaging'00 - PACS Design and Evaluation: Engineering and Clinical Issues, Vol. 3980, 2000, pp. 74-84.

[28] G. K. Anastassopoulos, A. N. Skodras, "JPEG2000 ROI Coding in Medical Imaging Applications," in: 2nd IASTED International Conference on Visualisation, Imaging and Image Processing, VIIP'02, Malaga, Spain, 2002, pp. 783-788.

[29] A. Albanese, J. Blömer, J. Edmonds, M. Luby, M. Sudan, "Priority Encoding Transmission," IEEE Trans. on Information Theory 42 (6) (1996) 1737-1744.

[30] J.K. Wolf, A.D. Wyner, J. Ziv, "Source coding for multiple description," Bell Syst. Tech. J. 59 (8) (1980) 1417-1426.

[31] J. Guédon, N. Normand, "The Mojette transform: the first ten yearsn," in: Proc. 12th International Conference on Discrete Geometry for Computer Imagery (DGCI), Vol. LNCS3429, Springer-Verlag, Poitiers, France, 2005, pp. 79-91.

[32] V. Goyal, "Muliple Description Coding: Compression Meets the Network," IEEE Signal Processing Magazine 18 (5) (2001) 74-93.

[33] B. Parrein, N. Normand, J. Guédon, "Multimedia Forward Error Correcting Codes," Annals of Telecommunications 58 (3-4) (2003) 448-463.

[34] B. Parrein, "Multiple Description of Information by Mojette Transform, PhD thesis (in french)," University of Nantes (November 2001).

[35] J. J. Lacan, V. Roca, S. Peltotalo, "Reed-Solomon Forward Error Correction (FEC)," draft-ietf-rmtbbfec- rs-02.txt (work in progress). Available at http://hal.inria.fr/docs/00/12/19/05/HTML/index.html.

[36] T. H. Oh, H. S. Lim, S. Y. Pang, "Medical Image Processing: from Lossless to Lossy Compression," Proc. IEEE TENCON'06 Region 10 Conference, (November 2005). 